\title{
New miRNA labeling method for bead-based quantification
}

\author{
Alberto Biscontin', Silvia Casara1', Stefano Cagnin', Lucia Tombolan¹, Angelo Rosolen², Gerolamo Lanfranchi*1 and \\ Cristiano De Pittà*1
}

\begin{abstract}
Background: microRNAs (miRNAs) are small single-stranded non-coding RNAs that act as crucial regulators of gene expression. Different methods have been developed for miRNA expression profiling in order to better understand gene regulation in normal and pathological conditions. miRNAs expression values obtained from large scale methodologies such as microarrays still need a validation step with alternative technologies.

Results: Here we have applied with an innovative approach, the Luminex ${ }^{\circ} \times M^{\circ} P^{\top m}$ technology validate expression data of differentially expressed miRNAs obtained from high throughput arrays. We have developed a novel labeling system of small RNA molecules (below $200 \mathrm{nt}$ ), optimizing the sensitive cloning method for miRNAs, termed miRNA amplification profiling (mRAP). The Luminex expression patterns of three miRNAs (miR-23a, miR-27a and miR-199a) in seven different cell lines have been validated by TaqMan miRNA assay. In all cases, bead-based meas were confirmed by the data obtained by TaqMan and microarray technologies.

Conclusions: We demonstrate that the measure of individual miRNA by the bead-based method is feasible, high speed, sensitive and low cost. The Luminex ${ }^{\circ} \mathrm{XMAP}^{\mathrm{TM}}$ technology also provides flexibility, since the central reaction can be scaled up with additional miRNA capturing beads, allowing validation of many differentially expressed miRNAs obtained from microarrays in a single experiment. We propose this technology as an alternative method to qRT-PCR for validating miRNAs expression data obtained with high-throughput technologies.
\end{abstract}

\section{Background}

MicroRNAs (miRNAs) are endogenous 18-24 nucleotides (nt) long noncoding RNAs (ncRNA) that control gene expression by targeting mRNAs and triggering either translation repression or degradation. The degree of complementarity between a miRNA and its mRNA target determines, at least in part, the regulatory mechanism [1]. Recently, a third less understood mechanism of small RNAs interference on gene expression involves heterochromatin silencing [2]. Many miRNAs are highly conserved among animals and plants [3] and it is estimated that up to $33 \%$ of all mRNA coding genes are negatively regulated by miRNAs $[4,5]$. miRNAs exhibit temporally and spatially regulated expression patterns during diverse developmental and physiological processes and clearly animals cannot survive without miRNAs [6,7]. Most of

*Correspondence: gerolamo.lanfranchi@unipd.it, cristiano.depitta@unipd.it Department of Biology and CRIBI Biotechnology Centre, Università degli Studi di Padova, Via U. Bassi, 58/B, 35121 Padova, Italy

Full list of author information is available at the end of the article the miRNAs that have been characterized so far in animals seem to regulate developmental processes, including larval stage transitions and neuronal development in C. elegans [8], growth control and apoptosis in Drosophila melanogaster [9] or haematopoietic differentiation in mammals. Many miRNAs have been found to display unique tissue [10], developmental stage [11] or diseasespecific patterns [12]. These observations imply that each tissue is characterized by a specific set of miRNAs that contribute to the definition of the features of that tissue. Hundreds of novel conserved and non-conserved microRNAs have been identified by bioinformatic analyses, suggesting that the total number of human miRNAs could reach 1,000 [13]. According to recent computational predictions, as many as 200 mRNAs can be regulated by a single miRNA, which implies that over one third of protein-coding genes in humans are regulated by miRNA [14,15].

So, the ability to monitor changes in miRNAs expression is important for understanding gene regulation both 
in physiological and pathological conditions. Several methodologies have been adapted for profiling miRNA expression: northern blotting with radiolabeled probes [16,17], cloning [18,19], massive parallel signature sequencing (MPSS) [20], quantitative PCR-based amplification of precursor [21] or mature miRNAs [22], SAGEbased techniques [23], bead-based profiling methods $[24,25]$ and oligonucleotide microarray [26]. Microarray technology has been successfully used for evaluating variations of miRNA expression during development $[27,28]$, differentiation [29], oncogenesis [30-32], disease progression $[33,34]$ and for the primary identification of new miRNAs that were predicted by bioinformatic approaches [35,36]. However, at present, no standard methodology exists for hybridization-based profiling of miRNAs and, as a consequence, comparison of expression data from different experiments can be difficult. To solve these problems it will be necessary to develop quality procedures for miRNA microarrays. Furthermore, miRNA expression obtained from high throughput arrays has to be validated with alternative technologies. Quantitative real-time PCR (qRT-PCR) has become the golden standard of miRNA quantification because it offers the highest sensitivity from small amounts of starting material and it is able to detect as less as 1-nt difference between miRNAs. Another interesting method is the Luminex ${ }^{\circ} \mathrm{xMAP}^{\mathrm{m}}$ system that is a multiplexed microsphere-based suspension array platform capable of performing and reporting up to 100 different analyses in a single reaction vessel $[37,38]$. In particular, oligonucleotide-capturing probes complementary to miRNAs of interest are coupled to carboxilated 5-micron polystirene beads impregnated with variable mixtures of two fluorescent dyes, each representing a single miRNA. Using this technique $\mathrm{Lu}$ and colleagues [24] were able to differentiate tumours that were instead inaccurately classified by mRNA profiles. Recently, a study performed with Luminex miRNA platform, identified new markers of human breast cancer subtype [39]. When compared to glass-slide microarrays, the bead-based miRNA arrays show many advantages such as easy of use, low cost, superior statistical performance, faster hybridization kinetics (solution hybridization) and higher flexibility in array preparation. Furthermore, the Luminex bead array system has been used in a wide range of multianalyte applications throughout the drug discovery and diagnostics fields and it is also widely adopted for quantitative multiplexed protein expression analysis [40,41].

In this study we have applied the Luminex $\mathrm{xMAP}^{\mathrm{m}}$ technology in a novel approach to validate expression data of differentially expressed miRNAs obtained from high throughput arrays. We have developed an innovative system for labeling of small RNA molecules (below 200 nt) optimizing the sensitive cloning method for miRNAs, termed mRAP, developed by Takada and Mano to define mouse miRNA transcriptional signature [19,42,43]. The expression patterns of three miRNAs (miR-23a, miR-27a and miR-199a) measured by our Luminex approach in seven different cell lines were validated by TaqMan miRNA assay. In all cases, the two technologies gave super imposable results. Our data demonstrate that beadbased detection of individual miRNA is a feasible approach, associated to high speed and low cost. The Luminex $\mathrm{xMAP}^{\mathrm{m}}$ technology is also feasible for multiplexing, since several beads prepared to capture different miRNAs can be added in the same reaction allowing the validation of many differentially expressed miRNAs obtained from large-scale approaches in a single experiment.

\section{Results and Discussion} miRNA expression in rhabdomyosarcoma cell lines

Rhabdomyosarcomas (RMS) are rare but very aggressive tumours of childhood that arise as a consequence of regulatory disruption of the growth and differentiation pathways of myogenic precursor cells [44]. Based on morphology, two major RMS subtypes can be identified: embryonal RMS (ERMS) and alveolar (ARMS). To better understand the global function of miRNA in RMS, we analyzed the expression profile of 7 different RMS cell lines (3 ARMS and 4 ERMS) using the mirVana miRNA Probe Set V1 (Ambion) that is a collection of about 400 amino-modified DNA oligonucleotides [45]. Briefly, the miRNA population from a single cell line was compared to a reference sample consisting of a pool of the seven small RNA samples ( $<200 \mathrm{nt}$ ) mixed in equal amounts. The miRNA microarray platform was able to distinguish PAX3-FKHR positive (RH4, RH30) and negative RMS (RD, CCA, SMS-CTR, RH36, RH18) cell lines through the expression pattern of about 120 miRNAs (data not shown). Since the translocation positive RMS patients fared worse than the negative counterpart [46] our results demonstrated the potential of miRNA expression profiling to classify different RMS subtypes, in agreement to previous gene expression studies [47-49], and set the basis for a further functional characterization of selected miRNAs implicated in RMS pathogenesis and in the different clinical behaviour and aggressiveness of the two RMS subtypes. We decided to study miRNAs with the greatest difference in expression between PAX3-FKHR positive and negative RMS. So expression levels for three discriminant miRNAs (miR-23a, miR-27a and miR-199a) were validated by $\mathrm{xMAP}^{\mathrm{mm}}$ technology and TaqMan qRTPCR.

\section{Testing the hybridization specificity and sensitivity between targets and capture probes coupled to microspheres}

To prepare the capture probes, 21-23 bases-long oligonucleotides with sequence complementary to each of the 
three differentially expressed miRNAs (miR-23a, miR-27a and miR-199a) in RMS cell lines, were synthesized and coupled to different color-coded microspheres in separate reaction tubes and then mixed for multiplexed assays. The targets were oligonucleotides complementary to capture probes and tagged with biotin at their 5 '-end. Capture probes (approximately 5,000 beads for each probe) were mixed in the same tube with targets at various amounts ranging from 15 amol to 300 fmol. Phycoerythrin (PE)-coniugated streptavidin was added to the reaction mixture to detect bound targets that were biotynilated. The signal of each target hybridized to its specific capture probe coupled to microspheres was determined by the fluorescence intensity of phycoerytrin. At least 100 microspheres of each set were analyzed by the Bio-Plex ${ }^{\mathrm{Tm}}$ system to obtain a median fluorescence intensity value (MFI) that was representative of the whole population of each set of beads. As shown in Figure 1A, the hybridization signal for miR-199a with capture probe varied with the amounts of added target in a logarithmic trend, reaching a plateau when the targets were present at the highest concentrations. Furthermore, the specificity of hybridization did not change while increasing from 1 to 3 the number of different microspheres in the reaction. We have evaluated the stability of conjugated microspheres at regular intervals during 80 days after conjugation. The hybridization signal is stable up to 35 days. As shown in Figure 1A, the hybridization signal for the miR-199a slightly decreased with the distance from conjugation, but the hybridization between the capture probe and the corresponding target remained specific and proportional. Furthermore, our data show that it is better to use microspheres conjugated in the same day to correctly detect miRNA expression values. We have also represented the curve in a log-scale (Figure 1B) highlighting the linearity range of our technique: the lower limit of sensitivity is $0.073 \mathrm{fmol}$ and the upper is $18.75 \mathrm{fmol}$. We have obtained comparable results with either miR-23a or miR-27a. We measured the specificity of our technique by testing the variation in hybridization signal intensity when miR-27a and miR-199a are captured with probes that contain one or two mismatches in their complementary sequences (see Table 1). We have evaluated the signal of each target (biotynilated oligonucleotides perfectly complementary to mature miRNA sequence) hybridized to the correspondent capture probes with perfect match, one or two mismatches. As described above, capture probes were mixed in the same tube with targets at various amounts (from 15 amol to $300 \mathrm{fmol}$ ). Figure 2 shows that for both miRNAs we obtain a significant decrease of hybridization fluorescence intensity with capture probes containing a single mismatch. The signal drop is even greater with two mismatches: $62 \%$ for miR-27a and $85 \%$ for miR-199a.
We have also determined the expression levels of above cited miRNAs, using the capture probes with perfect match, one or two mismatches in complex RNA populations obtained from seven RMS cell lines (RD, CCA, SMS-CTR, RH36, RH18, RH4, RH30) in comparison to a reference sample (Pool). As shown in Figure 3, we observe a decreasing trend in fluorescence signal for the capture probes with two mismatches. These experiments show that the specificity of a capture probe is proportional to the number of mismatches present in its sequence and that this specificity guarantee the correct quantification of miRNA expression levels in complex RNA populations.

\section{A new application of mRAP method for miRNA labeling}

We have developed a new sensitive miRNA labeling method based on the mRAP strategy recently developed by Mano and Takada for miRNA cloning [40,41]. This new procedure is described schematically in Figure 4. Small RNA molecules ( $<200-n t)$ purified by PureLink ${ }^{\mathrm{m}}$ miRNA Isolation Kit (Invitrogen) were polyadenylated with Poly(A) polymerase (PAP). Complementary DNAs corresponding to the miRNAs were then synthesized with the use of reverse transcriptase and a RT primer, named Oligo- $\mathrm{dT}_{15}-\mathrm{T} 7$, complementary to the poly(A) sequences added to the miRNA. This RT reaction was allowed for 30 minutes in order to synthesize cDNA molecules of about $200 \mathrm{nt}$. We have used a degenerated oligo(dT) to reduce the length of the neo-synthesized poly(A) tails to 15 nucleotides. Given that some reverse transcriptases possess terminal deoxynucleotidyl transferase activity, the synthesized cDNA strands frequently result with small poly $(C)$ overhangs at their 3 ' ends. After annealing a long 5'-adptor, named SMART-16attB1-T3, to such $\operatorname{poly}(\mathrm{C})$ overhangs, PCR was used to amplify the miRNA-derived cDNAs. Using a T3-biotinylated forward primer, we have obtained biotinylated cDNA that was detected with Phycoerythrin (PE)-coniugated streptavidin after hybridization reaction. Every step of this protocol was quality checked by Agilent Bioanalyzer 2100 and the resulting electropherograms are represented in Figure 4. Electropherogram analysis of the RT products have revealed two major bands of $\sim 30$ and $\sim 40$ nt that correspond to the 5'-adaptor (SMART-16attB1-T3) and the oligo- $\mathrm{dT}_{15}-\mathrm{T} 7$ primer respectively. The electropherograms also evidence the action of degenerated oligo(dT) that reduces the length of neo-synthesized cDNAs to 100-110 nucleotides by shortening the poly(A) to $15 \mathrm{nt}$ (Figure 4).

We have decided to block PCR reaction during the exponential phase at 22 cycles, to avoid distortion of the actual concentration of miRNAs in the sample under consideration. We have demonstrated that PCR cycles between 20 and 25 are sufficient to achieve a good level of 

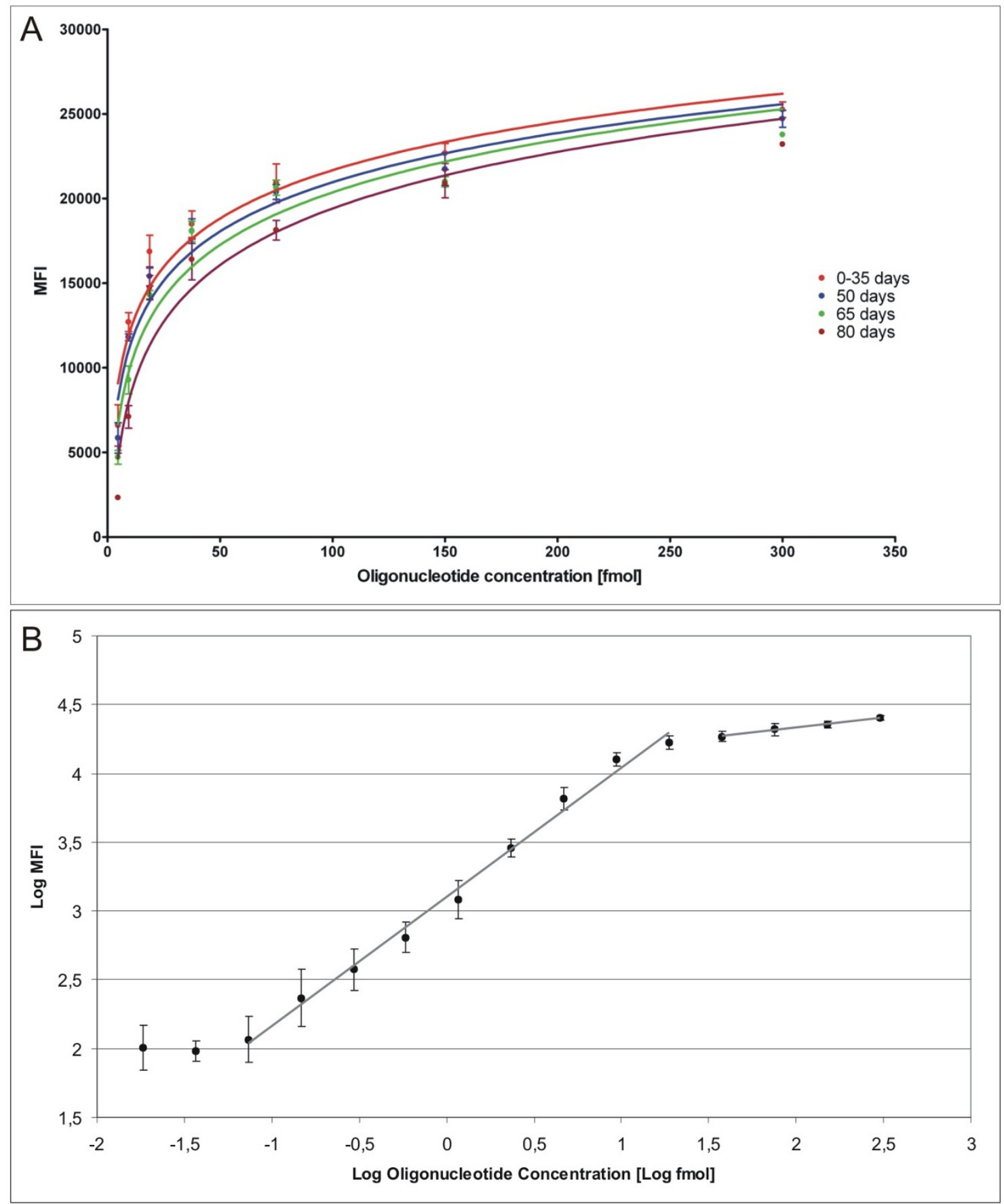

Figure 1 Stability of conjugated microspheres and sensitivity of hybridization. A) Increasing amounts of miR-199a target oligonucleotide (from $15 \mathrm{amol}$ to $300 \mathrm{fmol}$ ) show higher fluorescence levels reaching a plateau when the target is present at high concentrations. The curve shows a logarithmic trend. We observed a slightly decrease of the hybridization signal at 50, 65 and 80 days after conjugation. B) Calibration curve (0-35 days) of miR-199a in a log-scale from $15 \mathrm{amol}$ to $300 \mathrm{fmol}$. The two lines show the linear and plateau range of fluorescence levels representing by two different slopes: 0.94 and 0.15 respectively with $R^{2}=0.99$. The linearity range of our technique is comprised between a lower value of $0.073 \mathrm{fmol}$ and an upper value of $18.75 \mathrm{fmol}$. Mean value of expression and $95 \%$ confidence intervals are associated to each fluorescence value (MFI corresponds to Median Fluorescence Intensity). 


\begin{tabular}{lll}
\hline ID & Nucleotide sequence (5'-3') & Lenght (nt) \\
\hline miR-23a & GGAAATCCCTGGCAATGTGAT & 21 \\
miR-27a & GCGGAACTTAGCCACTGTGAA & 21 \\
miR-27a-1Mm & GCGGAACTAAGCCACTGTGAA & 21 \\
miR-27a-2MM & GCGGAAGTTAGCCACTGAGAA & 21 \\
miR-199a & GAACAGGTAGTCTGAACACTGGG & 23 \\
miR-199a-1MM & GAACAGGTAGTCTGAAGACTGGG & 23 \\
miR-199a-2MM & GAICAGGTAGTGTGAACACTGGG & 23 \\
Spike 18 & CATTGCACAATCAAGACTAAGA & 23 \\
\hline
\end{tabular}

Nucleotide sequence of capture probes synthesized with 5'-amino linker and a C12 spacer. Basepair mismatches (named MM) are in bold and underlined

amplification without reaching the plateau phase in which the expression differences would be invalidated (Figure 5A).

Finally, to verify the presence of miRNAs within the labeled cDNA population we have identified, by PCR, three specific miRNAs (miR-1, miR-206 and miR-450) using the sequence of each miRNA as forward primer, and the sequence for T7 promoter, which is common to all labeled molecules, as reverse primer. The result of PCR amplification of the three miRNAs is shown in Figure 5B.

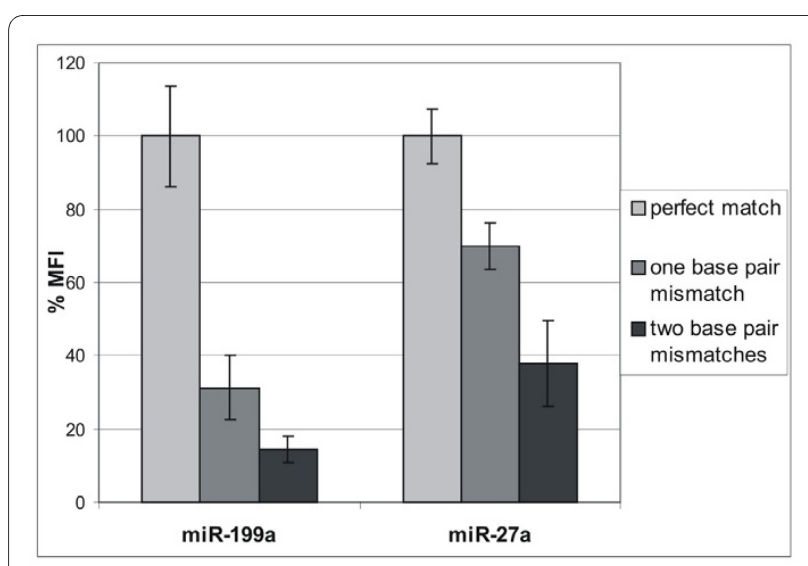

Figure 2 Specificity of hybridization between capture probes and miRNA targets. Biotynilated miR-199a and miR27a (bottom panel) were captured in solution by the corresponding probes with exactly matching sequences (light grey boxes) or with one (dark grey boxes) or two mismatched bases (black boxes). The fluorescence signal obtained by the hybridization of both the perfectly matching probes was set to $100 \%$. The hybridization signal between both capture probes and the corresponding miRNA target shows a statistically significant decrease ( $p$-value $<0.05$ ) when one or two mismatches are introduced into the capture sequence. Hybridization experiments were obtained with $18.75 \mathrm{fmol}$ of biotynilated targets that fall in the linearity range of our method as shown in Figure 1B. Mean value of expression and 95\% confidence intervals are associated to each fluorescence value (\%MFI corresponds to the percentage of Median Fluorescence Intensity).

\section{Validation of differentially expressed miRNAs by Luminex ${ }^{\circ}$ $\mathrm{XMAP}^{\mathrm{TM}}$ technology}

Using the $\mathrm{xMAP}^{\mathrm{mt}}$ assay described above we have determined the expression levels of miR-23a, miR-27a and miR-199a in seven RMS cell lines (RD, CCA, SMS-CTR, RH36, RH18, RH4, RH30) in comparison to a reference sample consisting of a pool of small RNA from each cell line mixed in equal amounts.

To evaluate the validity and feasibility of an assay, it is necessary to compare data with that obtained by other established technologies such as, in this case, microarray and qRT-PCR. As shown in Figure 6, the trends of expression levels measured for all the miRNAs by the three technologies (microarray, $\mathrm{xMAP}^{\mathrm{m}}$ and $\mathrm{qRT}-\mathrm{PCR}$ ) were very similar. Although the actual values of the relative miRNA hybridization signals in the seven RMS cell lines were not exactly the same for the three technologies, upregulated miRNAs (miR-23a and miR-27a) in PAX3FKHR positive RMS samples (RH4, RH30) detected originally by microarray were also up-regulated as detected by $\mathrm{xMAP}^{\mathrm{m}}$ and $\mathrm{qRT}-\mathrm{PCR}$, and signals for miRNAs downregulated in $P A X 3-F K H R$ negative RMS samples (RD, CCA, SMS-CTR, RH36, RH18) resulted consistently low by all three independent technologies. To better understand which are the techniques that provide the most similar results we have applied non-parametric Spearman correlation to the following paired comparisons for each tested miRNAs: $\mathrm{xMAP}_{\mathrm{TM}} v s$. microarray, $\mathrm{xMAP}_{\mathrm{TM}} v s$. qRT-PCR and qRT-PCR $v$. microarray. It is interesting to note that data obtained with $\mathrm{xMAP}^{\mathrm{ma}}$ technology seem to be more similar to those defined by microarray (Spearman correlation: $\mathrm{miR}-199 \mathrm{a}=0.64$, $\mathrm{miR}-23 \mathrm{a}=0.64$, miR$27 \mathrm{a}=0.84)$ respect to qRT-PCR (Spearman correlation: miR-199a $=0.67, \operatorname{miR}-23 \mathrm{a}=0.53$, miR-27a $=0.81$ ). From this comparison we could observe that the PCR amplification step introduced in $\mathrm{xMAP}^{\mathrm{mx}}$ labeling method does not affect miRNA expression levels whereas miRNA expression data obtained from $<200$-nt RNA molecules 


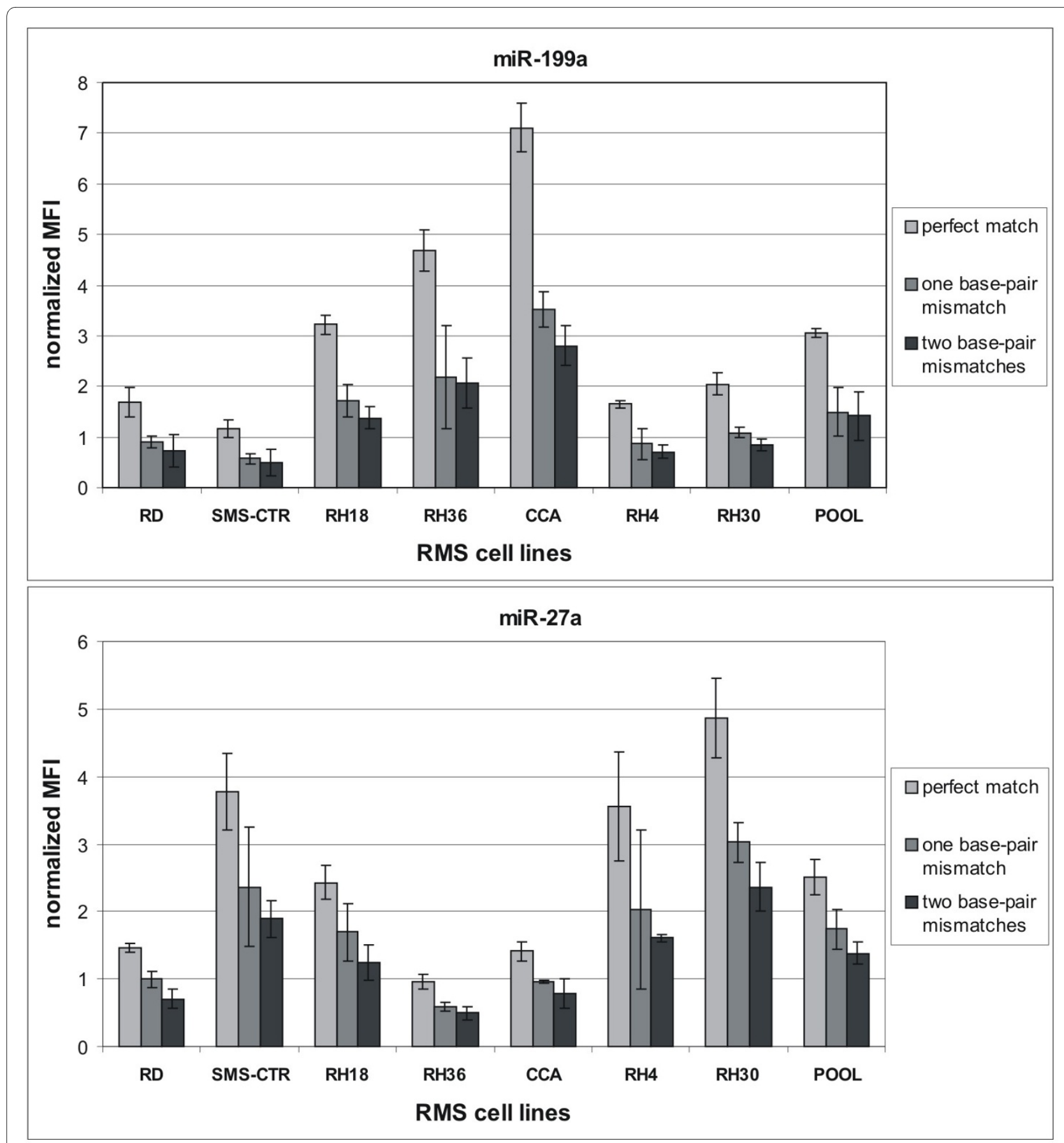

Figure 3 Specificity of hybridization between capture probes and miRNA targets in complex RNA populations. miR-199a (top panel) and miR27a (bottom panel) were captured from labeled total RNA populations prepared from 7 different RMS cell lines (RD, SMS-CTR, RH18, RH36, CCA, $\mathrm{RH} 4, \mathrm{RH} 30$ ) and a pool these RNA preparations mixed in equal amounts (POOL) with capture probes with exactly matching sequences (light grey boxes) or with one (dark grey boxes) or two mismatched bases (black boxes). The hybridization signal in all complex RNA mixtures between both capture probes and the corresponding miRNA target in comparison to the reference pooled sample shows a statistically significant decrease ( $p$-value $<0.05$ ) when one or two mismatches are introduced into the capture sequence. Mean value of expression and $95 \%$ confidence intervals are associated to each fluorescence value (normalized MFI represents the average of the ratio between the value of fluorescence of miRNAs and the spike).

(microarray and $\mathrm{xMAP}^{\mathrm{m}}$ ) differ slightly from those obtained from total RNA (qRT-PCR). This is particularly evident by non-parametric Spearman correlation for qRT-PCR $v s$. microarray $(\mathrm{miR}-199 \mathrm{a}=0.94$, miR-23a $=$ 0.42 , miR-27a $=0.70$ ) with the exception of miR-199a.

\section{Conclusions}

We have described a new method that makes use of $\mathrm{xMAP}^{\mathrm{m}}$ technology for the quantitative determination of single miRNAs. This approach quantifies miRNA expression levels based on hybridization of small RNA popula- 


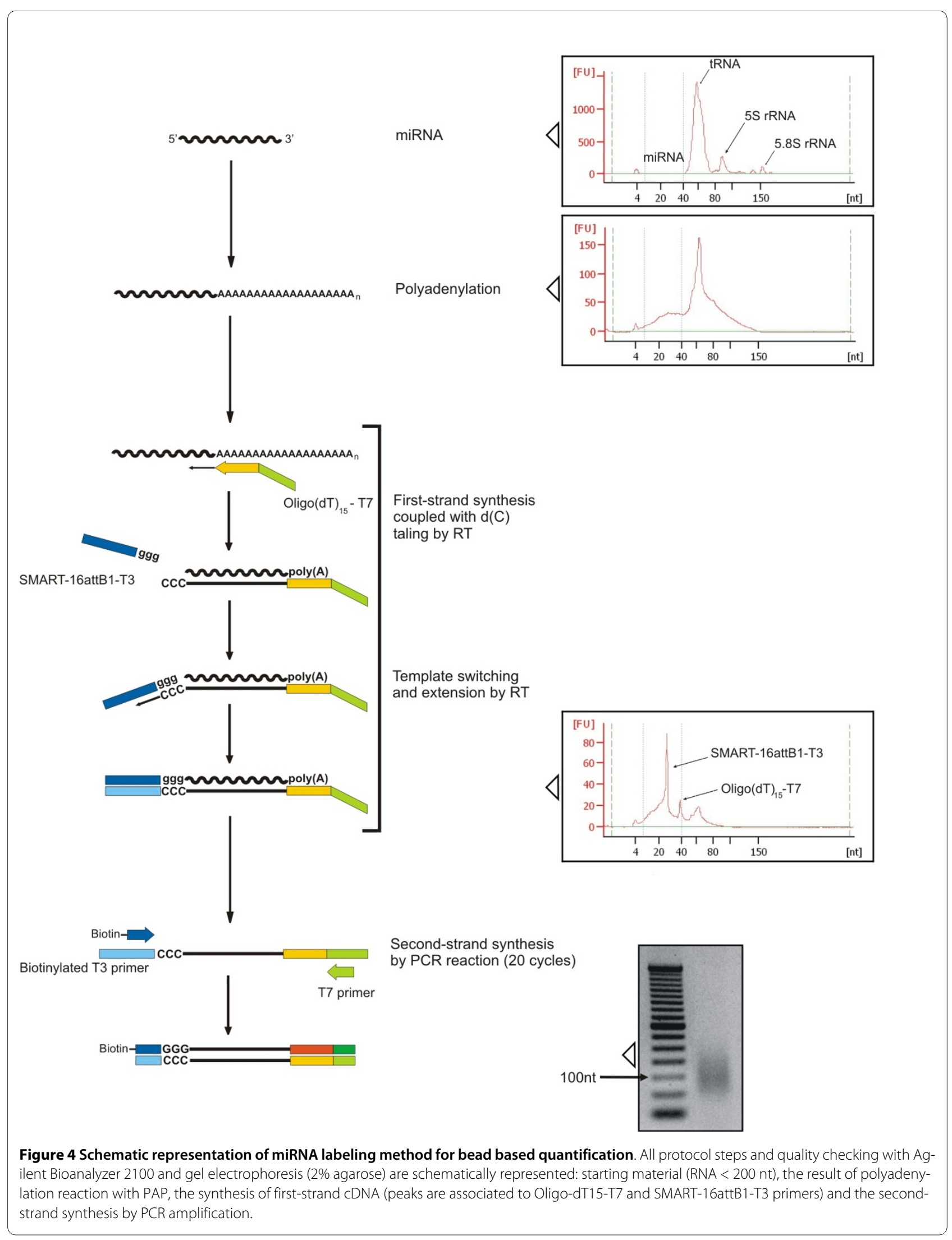




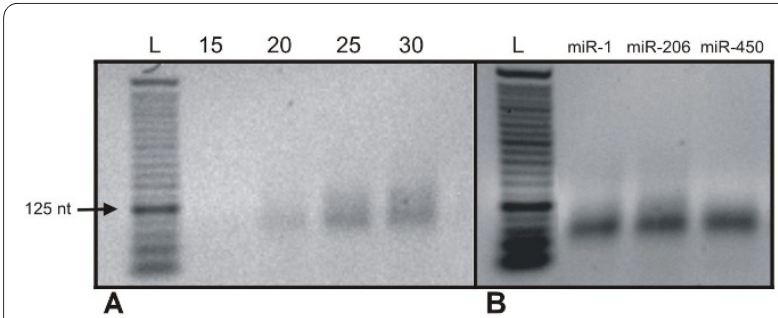

Figure 5 Second-strand synthesis by PCR reaction. A)Determination of the optimal PCR amplification cycle: the $2 \%$ agarose gel shows the amplification products of RH30 first-strand CDNA stopped at 15,20, 25 and 30 PCR cycles. B) Presence of specific miRNAs in the labeled CDNA population: the $2.25 \%$ agarose gel shows PCR amplification products of three specific miRNAs (miR-1, miR-206 and miR-450) performed by using the sequence of each miRNA as forward primer, and the sequence for T7 promoter, which is common to all labeled molecules, as reverse primer.

tions to the probes designed for miRNAs of interest that are coupled to different color-coded microspheres. A flow-cytometer is used to simultaneously measure the hybridization signal associated with the surface of the microspheres and to categorize the color-coded microspheres. The method allows to obtain up to 100 independent measures. This $\mathrm{xMAP}^{\mathrm{m}}$ method offers affordable cost, speed, and high flexibility with capability for multiplexed assays that are increasingly needed. It is valuable for applications such as diagnostic detection of disease miRNAs from clinical samples and validation of differentially expressed miRNAs obtained from microarray analysis.

We have evaluated the stability of conjugated microspheres demonstrating that they are stable for about one month. We have also tested the specificity of hybridization between targets and capture probes showing that the introduction of a single mismatch in the sequence of capture probes significantly hampers its hybridization capacity (Figures 2 and 3). These data have demonstrated the specificity of our capture probes to correctly identify and quantify the expression levels of miRNAs. We have developed a new sensitive small RNAs labeling protocol introducing some changes on mRAP strategy for miRNA cloning. This protocol has been applied successfully to validate the expression levels of three miRNAs that were found differentially expressed in positive and negative $P A X 3 / F K H R$ alveolar rhabomyosarcomas by microarray experiments. To demonstrate the reliability of this method, the transcriptional levels of these miRNAs were also determined by qRT-PCR with TaqMan probes. The most significant information which emerges comparing the data obtained by the three different technologies (microarray, $\mathrm{xMAP}^{\mathrm{m}}$ and $\mathrm{qRT}-\mathrm{PCR}$ ) is represented by the general overlapping of the expression profiles obtained. It is interesting to note that small differences in miRNAs expression data could depend on the approaches used for total RNA purification rather than on different labeling protocols. In fact, microarray and $\mathrm{xMAP}^{\mathrm{Tw}}$ data obtained from labeling < 200-nt RNA molecules, are more similar compared to qRT-PCR data obtained from labeling total RNA, as demonstrated by the Spearman non parametric correlation.

We think that the $\mathrm{xMAP}^{\mathrm{m}}$ technology associated with our new labeling protocol could become an alternative and equally reliable method in the study of expression of a limited number of miRNAs respect to qRT-PCR with TaqMan probes. The comparison of the two experimental approaches (see Table 2) shows that the $\mathrm{xMAP}^{\mathrm{su}}$ technique is less expensive and more flexible allowing the simultaneous analysis of a larger number of miRNAs from the same sample. In addition, $\mathrm{xMAP}^{\mathrm{ma}}$ does not need for specific reverse transcription reactions for each miRNA and provides at least 100 independent measures for each miRNA, improving the statistical power. Moreover, $\mathrm{xMAP}^{\mathrm{mi}}$ expression data can be normalized respect to a spike fluorescence signal added in known quantities in the early stages of the labeling reaction, allowing the control over all stages of the reaction. In this way there is no need of an endogenous control, like in qRT-PCR, whose representativeness is sample-dependent.

Probably, a negative aspect of $\mathrm{xMAP}^{\mathrm{m}}$ technology is represented by the use of enriched total RNA ( $<200 \mathrm{nt}$ ) that requires higher amounts of starting material respect to qRT-PCR. To avoid the enrichment step of $<200 \mathrm{nt}$ RNA molecules, we propose the use of LNA captureprobe oligonucleotides that increase the affinity of the oligonucleotide for its complementary RNA target leading to a significant enhance in stability and specificity of the duplex.

We think that the technology we have developed could be an alternative method to qRT-PCR for validating miRNAs expression data obtained with a large scale technology such as microarray and it could have a wide application in clinical, pharmaceutical, agricultural and environmental studies.

\section{Methods}

\section{Cell culture}

Human alveolar RMS (ARMS) cell lines, positive for PAX3-FKHR translocation (RH4, RH30), negative for PAX3-FKHR translocation (RH18) and human embryonal RMS (ERMS) cells (RD, RH36, CCA, SMS-CTR) were maintained in modified Eagle's medium (DMEM) containing $10 \%$ fetal calf serum, penicillin $(100 \mathrm{U} / \mathrm{ml})$, and streptomycin $(100 \mu \mathrm{g} / \mathrm{ml})$ (Invitrogen) at $37^{\circ} \mathrm{C}, 5 \% \mathrm{CO} 2 \mathrm{in}$ a humidified incubator.

The human RMS cell lines RH30 and RD were purchased from ATCC (Manassas, VA); RH4 and RH18 were a gift of Dr P.J. Houghton (St Jude Children's Hospital, 


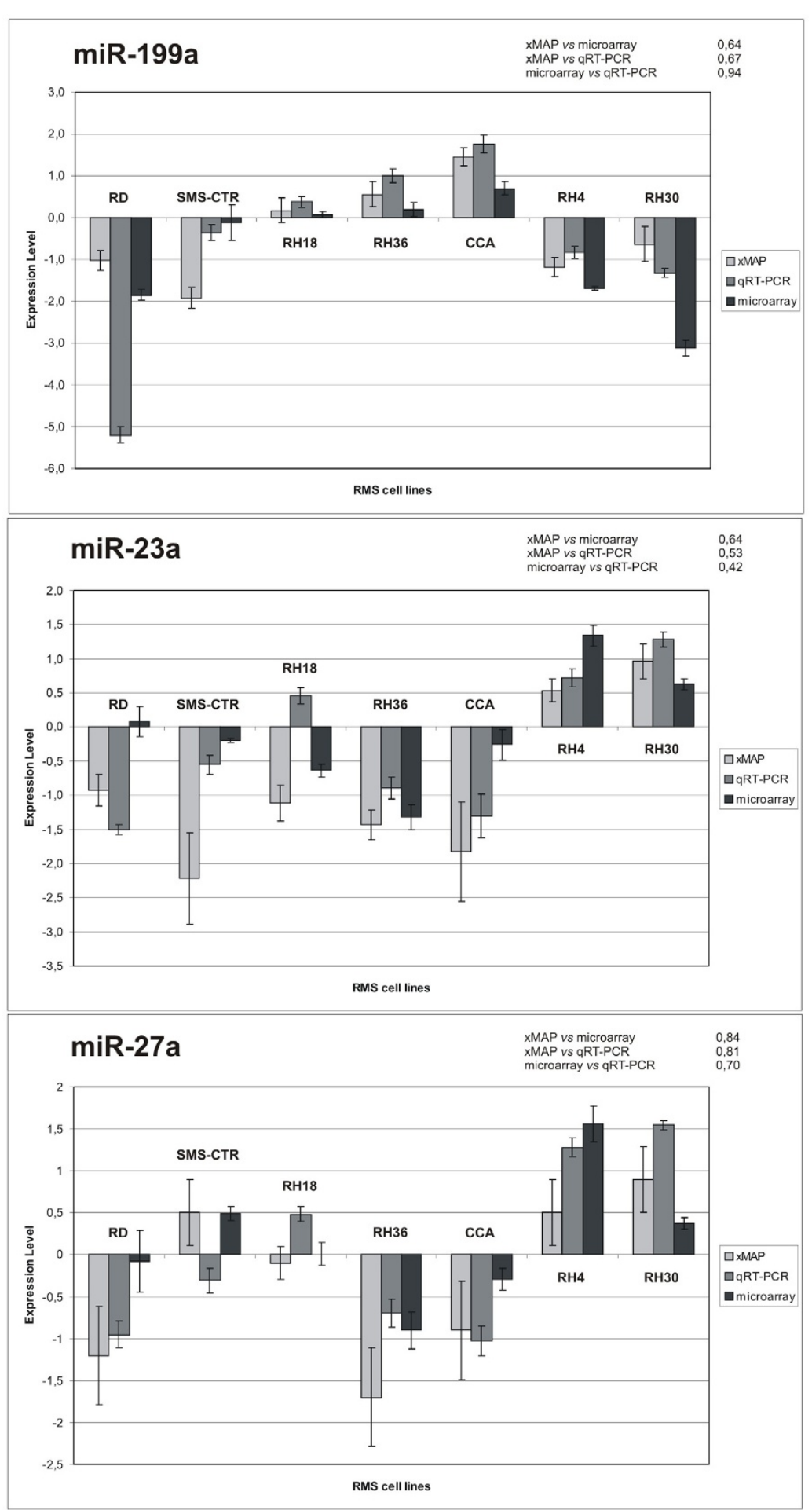

Figure $\mathbf{6}$ Comparison of three different techniques used to define miRNA expression profiles in seven RMS cell lines. Expression levels of miR199a, miR-23a and miR-27a in seven RMS cell lines obtained with three methods (XMAPTM, qRT-PCR and microarray) are represented. Mean value of expression and $95 \%$ confidence intervals are associated to each miRNA. The Figure also indicate, for each tested miRNAs, non-parametric Spearman correlations for the following paired comparisons: $X M A P^{\text {TM }}$ vs. microarray, XMAP ${ }^{T M}$ vs. QRT-PCR and qRT-PCR vs. microarray. 
Table 2: Comparison of XMAP ${ }^{\mathrm{Tm}}$ and qRT-PCR techniques

\begin{tabular}{llc}
\hline & XMAP'm & qRT-PCR \\
\hline$<200$ nt RNA molecules enrichment & YES & NO \\
RT step & YES & YES \\
Amplification & YES & YES \\
Endogenous control & NO & YES \\
Control spike & YES & NO \\
Multiplexing & YES & NO \\
Liquid-phase hybridization & YES & NO \\
No. of measures & 100 & 1 \\
Custom-tailored assay & YES & NO \\
Cost & LOW & High \\
\hline
\end{tabular}

Memphis, TN); SMS-CTR, RH36, CCA were obtained from Dr M. Tsokos (NCI, Bethesda, MD).

\section{Isolation of small RNA molecules}

Total RNA was prepared from seven cell lines of human rhabdomyosarcoma using a modified TRIzol (Life Technologies Corporation, Carlsbad, CA, USA) protocol for small RNA enrichment. A pellet of about $6 \times 10^{6}-9 \times 10^{6}$ cells was dissolved in $1 \mathrm{ml}$ of TRIzol and the supernatant, containing total RNA, was purified by PureLink ${ }^{\mathrm{TM}}$ miRNA Isolation Kit (Life Technologies Corporation) that was specifically designed to enrich total RNA preparation for $<$ 200-nt RNA molecules. RNA quantity and quality were assessed by Nanodrop (NanoDrop Technologies, Wilmington, DE, USA) spectrophotometry and microelectrophoresis using Small RNA Nano LabChip by Agilent 2100 bioanalyzer (Agilent Technologies, Palo Alto, CA, USA) respectively.

miRNA expression profiling and statistical analysis of data microRNA expression profiling was carried out using the "mirVana Probe Set V1" (Ambion) that is a collection of about 400 amine-modified DNA oligonucleotides representing a panel of the human, mouse and rat microRNAome in the miRNA Registry (miRBase - Release 9). The probes are 42-46 nucleotides (nt) long, with 18-24 nt segment targeting a specific miRNA, and the remaining sequence serving as spacer. We analyzed the expression profiles of 7 different rhabdomyosarcoma cell lines: 3 ARMS (RH4, RH30, RH18) and 4 ERMS (RD, RH36, CCA, SMS-CTR). The miRNA population from each cell line was compared to a reference sample consisting of a pool of the 7 total RNA samples mixed in equal amounts. Two replicates of each experiment were performed using different microarray slides, in which sample and reference RNAs, labeled either with Cy3 or Cy5 fluorochromes, were crossed in both combinations (dyeswapping procedure). miRNAs were labeled with the
mirVana Labeling Kit (Ambion) and amine-reactive dyes (GE Healthcare) as recommended by the manufacturer's protocol [45]. Normalization of expression levels of all spot replicates was performed by MIDAW [50]. Principal component analysis, cluster analysis and profile similarity searching were performed with tMev software [51]. One and two class Significance Analysis of Microarray (SAM) allowed to identify differentially expressed miRNAs [52].

\section{Capture probe and its coupling to microspheres}

A sequence of 21-23 nt complementary for each tested miRNAs (listed in the Table 1) was chosen as capture probe and synthesized with 5 -amino linker and a C12 spacer (PRIMM, Milan, Italy). Capture-probe oligonucleotides were covalently linked to carboxylated fluorochrome microspheres (Bio-Rad Laboratories, Hercules, CA, USA) in water-soluble carbodiimide. Specifically, $1 \times$ $10^{6}$ carboxylated microspheres were pelleted in a microcentrifuge for 5 minutes at $12,000 \times \mathrm{g}$ and then supernatant was carefully removed. The dry microspheres were dissolved in $20 \mu \mathrm{l}$ of a buffer containing $0.1 \mathrm{M}$ MES (Sigma-Aldrich, St. Louis, MO, USA) at $\mathrm{pH}$ 4.5. The amino-substituted capture probe was dissolved in molecular biology grade water at a concentration of $100 \mu \mathrm{M}$ and $0.5 \mu \mathrm{l}$ of the solution (containing 0.05 nmole of capture probe oligonucleotides) was added to the beads for the coupling reaction. The coupling reaction was performed by adding $2.5 \mu \mathrm{l}$ of a freshly made solution of 10 $\mathrm{mg} / \mathrm{ml}$ 1-ethyl-3-(3-dimethylaminopropyl) carbodiimide hydrochloride (EDC) (Pierce, Thermo Scientific, Wilmington, DE, USA) in molecular biology grade water. The mixture of microspheres, capture probes, and EDC was vortexed briefly and incubated at room temperature for 30 minutes in the dark. Occasionally, the reaction was mixed by finger flicking the tube to keep the microspheres in suspension. A second incubation steps was done adding a freshly-made solution of $10 \mathrm{mg} / \mathrm{mL}$ EDC in molecular biology grade water. After the coupling reac- 
tion, $500 \mu \mathrm{l}$ of $0.02 \%$ Tween 20 (Sigma-Aldrich) was added to the microspheres. The solution was mixed well by vortex and centrifuged for 6 minutes at 12,000×g. The supernatant containing free-capture-probe oligonucleotides and excess EDC was carefully removed. The coupled microspheres were washed in $500 \mu \mathrm{l}$ of $0.1 \%$ SDS (Sigma-Aldrich) by vortex and centrifuged for 5 minutes at $12,000 \times \mathrm{g}$. Finally, the supernatant was removed and the capture-probe conjugated microspheres were resuspended in $20 \mu \mathrm{l}$ of TE $\mathrm{pH} 8.0$ and stored at $4^{\circ} \mathrm{C}$ in a dark box (stable for at least 6 months). The microspheres were diluted in TE buffer and counted using a Bürker chamber under the microscope at $100 \times$ magnification.

\section{miRNA labeling}

At the beginning, $1 \mu \mathrm{g}$ of small RNA molecules $(<200-\mathrm{nt})$ and $200 \mathrm{pg}$ of a synthetic pre-labeling control RNA (5'UCUUAGUCUUGAUUGUGGCAAUG-3', PRIMM) were mixed in order to control target preparation efficiency and to normalize expression data. The mixture was polyadenylated using Poly(A) Tailing Kit (Ambion) according to the manufactures' instructions. The reaction was precipitated with $\mathrm{NaOAc} 3 \mathrm{M} \mathrm{pH} 5.5$ (1/10 volume) and absolute ethanol ( 4 volumes) overnight at $-20^{\circ} \mathrm{C}$. The polyadenylated RNA molecules were resuspended in 15 $\mu \mathrm{l}$ of $\mathrm{H}_{2} \mathrm{O}$ RNase free and then volume was reduced to 3.2 $\mu \mathrm{l}$ by vacuum (VR-1, Heto-Holten, Denmark). miRNAs labeling was performed by mRAP modified protocol in which miRNA-derived cDNAs were flanked by synthesized oligomers at each end. The SMART (switching mechanism at the 5'-end of RNA templates of reverse transcriptase) oligo sequence (SMART-16attB1-T3: 5'TACAAAAAAGCAGGCTAATTAACCCTCAC-

TAAAggg-3') and the overhang of the oligo- $\mathrm{dT}_{15}$-T7 primer (5'-GTGAATTGTTAATACGACTCACTATAGGCGC $\left.[\mathrm{dT}]_{15} \mathrm{~N}-3^{\prime}\right)$ were used for first strand synthesis. First strand cDNA synthesis was performed from $500 \mathrm{ng}$ of small RNA in a $10 \mu \mathrm{l}$ reaction. Then, the reaction was diluted $1: 2$ and incubated at $72^{\circ} \mathrm{C}$ for $7 \mathrm{~min}$. Second strand reaction mix was added to $3.0 \mu \mathrm{l}$ of diluted first strand cDNA to give a final concentration of $1 \mathrm{X} \mathrm{BD}$ Advantage 2 PCR reaction buffer (Clontech Laboratories, Mountain View, CA, USA), $0.2 \mathrm{mM}$ dNTPs, $100 \mathrm{nM}$ primers (T3-biotinylated forward primer: 5 ' - biotAATTAACCCTCACTAAAGGG-3' and T7 reverse primer: 5'- TAATACGACTCACTATAGG-3') and 1X of Advantage 2 DNA polymerase mix (Clontech Laboratories) in a total volume of $25 \mu \mathrm{l}$. This second strand reaction mixture was incubated for 22 cycles of the following steps: 15 $\sec 95^{\circ} \mathrm{C}, 20 \sec 51^{\circ} \mathrm{C}$ and $20 \sec 72^{\circ} \mathrm{C}$. Only those ss cDNAs having a SMART anchor sequence at the 5 '-end were used as template and exponentially amplified. The second strand reaction was precipitated in sodium acetate-ethanol solution and dissolved in $11 \mu \mathrm{l}$ TE buffer $\mathrm{pH}$
8.0 (10 mM TrisHCl pH 8.0, 1 mM EDTA). Biotinylated cDNA quantity was assessed by Nanodrop spectrophotometer (Nanodrop Technologies) and stored at $-20^{\circ} \mathrm{C}$ until hybridization with microspheres. Labelled target produced by a single PCR reaction was sufficient for two hybridization reactions.

\section{Hybridization of targets to capture probes coupled to microspheres}

The microspheres of each probe set were resuspended by vortexing for approximately 20 seconds. A microsphere mixture was prepared by diluting coupled stocks to 150 microspheres of each set/ $\mu \mathrm{l}$ in 1.5× TMAC Hybridization Buffer (5 M tetramethylammonium chloride, $0.15 \%$ Sarkosyl; $75 \mathrm{mM}$ Tris- $\mathrm{HCl} \mathrm{pH}$ 8.0; and $6 \mathrm{mM}$ EDTA, pH 8.0), followed by vortex mixing for approximately $20 \mathrm{sec}-$ onds. Two $\mu \mathrm{g}$ of biotinylated DNA target in $17 \mu \mathrm{L}$ of TE buffer $\mathrm{pH} 8.0$ was added to $33 \mu \mathrm{l}$ of microsphere mixture (approximately 5,000 beads per color) in the wells of a 96well plate. $17 \mu \mathrm{l}$ of TE buffer $\mathrm{pH} 8.0$ were added to background wells. Each well reaction was mixed gently by pipetting up and down several times and the labeled DNA was denatured by heating at $95-100^{\circ} \mathrm{C}$ for $3 \mathrm{~min}$. The hybridization mixture was incubated at $48^{\circ} \mathrm{C}$ for 17 $\mathrm{h}$, covering the plate to prevent evaporation, in a Eppendorf microplate incubator with shaking speed of $700 \mathrm{rpm}$. After incubation, the hybridization mixture was spun down for $3 \mathrm{~min}$ at $3,000 \times \mathrm{g}$ to pellet the microspheres. Supernatant was carefully removed with a pipette without disturbing the microspheres. During centrifugation, fresh reporter mix was prepared by diluting streptavidinconjugated R-phycoerythrin (Invitrogen) to $3 \mathrm{mg} / \mathrm{ml}$ in $1 \times$ TMAC Hybridization Buffer and $75 \mu$ of reporter mix were added to the microspheres. The solution was gently mixed by pipetting and incubated in the dark at $48^{\circ} \mathrm{C}$ for 15 minutes in a Eppendorf microplate incubator. $50 \mu \mathrm{l}$ of each sample were transferred to a Multiscreen HTS plate (Millipore) and analyzed on the BioPlex ${ }^{\mathrm{mm}}$ (Luminex $100^{\mathrm{mm}}$, BioRad) machine at hybridization temperature.

\section{Bead-based detection}

Each set of microspheres was distinguished by assigned colour code (different percentage of red and orange) inside the microspheres. In our experiments we have used four different probe sets (regions: 1, 21, 51 e 57). The fluorescence associated to the surface of each bead, corresponding to the amounts of bound miRNAs, was detected and measured by the laser detector. The BioPlex $^{\mathrm{mm}}$ (Luminex $100^{\mathrm{m}}$, Bio-Rad Laboratories) system detects fluorescent dyes with an excitation wavelength of $-532 \mathrm{~nm}$ and emission wavelength $-580 \mathrm{~nm}$. For each experiment, 100 events of each subset of microspheres were analyzed on the Bio-Plex ${ }^{\text {ma }}$ system to obtain a 
median fluorescence intensity value (MFI) that was representative of the whole population of each set of beads.

\section{Computational analyses (data processing and quality control)}

To eliminate bead-specific background, the reading of every bead for every samples was first processed by subtracting the average readings of that particular bead in the absence of target miRNAs. Samples with median fluorescence intensity values smaller than background signals were removed. Every samples was assayed in three wells. Each of the three wells contained 4 probes: miR23a, miR-27a, miR-199a and one pre-labeling control (Spike-18). Expression data were scaled according to the pre-labeling control in order to normalize readings from different probe/bead sets for the same sample and to normalize for the labeling efficiency. Technical replicate samples for each probe were summarized by their mean profile and expression data (test/control) were log2 transformed. The error associated to each probe is obtained by quadratic propagation from standard deviation.

\section{qRT-PCR TaqMan}

TaqMan MicroRNA Assays incorporate a target-specific stem-loop, reverse transcription primer. The stem-loop structure provides specificity only for the mature miRNA target and forms a RT primer/mature miRNA-chimera that extends the 5 '-end of the miRNA. The resulting longer RT amplicon presents a template amenable to standard real-time PCR using TaqMan Assays [22]. In brief, according to the manufacture's instructions (Applied Biosystems), each $15 \mu \mathrm{l}$ RT reaction contained purified $10 \mathrm{ng}$ of total RNA, $3.0 \mu \mathrm{l}$ of $5 \times$ stem-loop RT primer, $1 \times \mathrm{RT}$ buffer, $0.25 \mathrm{mM}$ each of dNTPs, $50 \mathrm{U}$ MultiScribe ${ }^{\mathrm{m} x}$ reverse transcriptase and $3.8 \mathrm{U}$ RNase inhibitor. The reactions were incubated in a Mastercycler EP gradient $S$ (Eppendorf) in $0.2 \mathrm{ml} \mathrm{PCR}$ tubes for $30 \mathrm{~min}$ at $16^{\circ} \mathrm{C}, 30$ min at $42^{\circ} \mathrm{C}$, followed by $5 \mathrm{~min}$ at $85^{\circ} \mathrm{C}$, and then held at $4^{\circ} \mathrm{C}$. RT products were diluted two times with $\mathrm{H}_{2} \mathrm{O}$ prior to setting up PCR reaction. Each real-time PCR for each miRNA assay (10 $\mu$ l volume) was carried out in triplicate, and each $10 \mu \mathrm{l}$ reaction mixture included $1 \mu \mathrm{l}$ of diluted RT product, $5 \mu \mathrm{l}$ of $2 \times$ TaqMan $^{\circ}$ Universal PCR Master

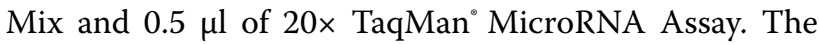
reaction was incubated in a 7500 Real-Time PCR System (Applied Biosystems) in 96- well plates at $95^{\circ} \mathrm{C}$ for $10 \mathrm{~min}$, followed by 40 cycles of the following steps: $95^{\circ} \mathrm{C}$ for 15 sec and $60^{\circ} \mathrm{C}$ for $1 \mathrm{~min}$. The threshold cycle (CT) is defined as the fractional cycle number at which the fluorescence exceeds the fixed threshold of 0.2 . To evaluate differences in miRNA expression, a relative quantification method was chosen where the expression of the miRNA target is standardized by a non-regulated small non-coding RNA used as reference.
Consequently, three replicates of each sample and endogenous control were amplified. U6B small nuclear (RNU6B) was used as endogenous control because the level of this small RNA remains essentially constant from sample to sample. To calculate the relative expression ratio, the $2^{-\Delta \Delta C t}(R Q$, relative quantification) method implemented in the 7500 Real Time PCR System software [53] was used. This method determines the change in expression of a nucleic acid sequence (target) in a test sample relative to the same sequence in a calibrator sample.

\section{Authors' contributions}

$A B$ performed small RNA molecules isolation, capture probes coupling to microspheres, miRNA labeling method, bead-based detection with BioPlex and computational analyses (data processing and quality control). SC performed RMS cell lines culture, small RNA molecules isolation, microarray experiments and QRT-PCR validation. SC participated in conceiving the study and in development of miRNA labelling method. LT performed RMS cell lines culture and participated in total RNA extraction. AR provided RMS cell lines and revised the manuscript. GL supervised the study, participating in the design and coordination of the work, the interpretation of the results and revision of the manuscript. CDP conceived and supervised the study, participating in the design and coordination of the work, the interpretation of data and manuscript writing. All Authors read and approved the final version of the manuscript declaring that they have no potential conflicts of interests.

\section{Acknowledgements}

This work was supported by Associazione Italiana per la Ricerca sul Cancro (AIRC) and Biotech Action III bis (CIPE 3/06 DGR 4073 19/12/2006 Veneto Region). The authors wish to thank Emanuele Papini, Paola Cecchini and Regina Tavano (Dept. Biomedical Sciences and C.R.I.B.I. Biotechnology CentreUniversity of Padova, Italy) for technical support in bead-based detection with BioPlex. We are also grateful to Angelica Zin for RMS cell lines.

\section{Author Details}

1Department of Biology and CRIBI Biotechnology Centre, Università degli Studi di Padova, Via U. Bassi, 58/B, 35121 Padova, Italy and 2Clinica di

Oncoematologia Pediatrica, Azienda Ospedaliera-Università degli Studi di Padova, Vi Giustiniani 3, 35128 Padova, Italy

Received: 21 December 2009 Accepted: 16 June 2010

Published: 16 June 2010

\section{References}

1. Engels BM, Hutvagner G: Principles and effects of microRNA-mediated post-transcriptional gene regulation. Oncogene 2006, 25:6163-6169.

2. Lippman Z, Martienssen R: The role of RNA interference in heterochromatic silencing. Nature 2004, 431:364-370.

3. Zhang $B$, Wang $Q$, Pan $X$ : MicroRNAs and their regulatory roles in animals and plants. J Cell Physiol 2007, 210:279-289.

4. Harfe BD: MicroRNAs in vertebrate development. Curr Opin Genet Dev 2005, 15:410-415.

5. Xi Y, Shalgi R, Fodstad O, Pilpel Y, Ju J: Differentially regulated microRNAs and actively translated messenger RNA transcripts by tumor suppressor p53 in colon cancer. Clin Cancer Res 2006, 12:2014-2024.

6. Bartel DP: MicroRNAs: genomics biogenesis, mechanism, and function. Cell 2004, 116:281-297.

7. Stefani G, Slack FJ: Small non-coding RNAs in animal development. Nat Rev Mol Cell Biol 2008, 9:219-230.

8. Lee RC, Ambros V: An extensive class of small RNAs in Caenorhabditis elegans. Science 2001, 294:862-864.

9. Jaubert S, Mereau A, Antoniewski C, Tagu D: MicroRNAs in Drosophila: the magic wand to enter the Chamber of Secrets? Biochimie 2007, 89:1211-1220.

10. Landgraf P, Rusu M, Sheridan R, Sewer A, lovino N, Aravin A, Pfeffer S, Rice A, Kamphorst AO, Landthaler M, Lin C, Socci ND, Hermida L, Fulci V, Chiaretti S, Foà R, Schliwka J, Fuchs U, Novosel A, Müller RU, Schermer B, 
Bissels U, Inman J, Phan Q, Chien M, Weir DB, Choksi R, De Vita G, Frezzetti D, Trompeter HI, et al:: A mammalian microRNA expression atlas based on small RNA library sequencing. Cell 2007, 129:1401-1414.

11. Pasquinelli $A E$, Ruvkun G: Control of developmental timing by microRNAs and their targets. Annu Rev Cell Dev Biol 2002, 18:495-513.

12. Rana TM: I lluminating the silence: understanding the structure and function of small RNAs. Nat Rev Mol Cell Biol 2007, 8:23-36.

13. Bentwich I, Avniel A, Karov Y, Aharonov R, Gilad S, Barad O, Barzilai A, Einat $P$, Einav U, Meiri E, Sharon E, Spector Y, Bentwich Z: Identification of hundreds of conserved and nonconserved human microRNAs. Nat Genet 2005, 37:766-770

14. Lewis BP, Shih IH, Jones-Rhoades MW, Bartel DP, Burge CB: Prediction of mammalian microRNA targets. Cell 2003, 115:787-798.

15. Wang $X$, El Naqa IM: Prediction of both conserved and nonconserved microRNA targets in animals. Bioinformatics 2008, 24:325-332.

16. Reinhart BJ, Slack FJ, Basson M, Pasquinelli AE, Bettinger JC, Rougvie AE, Horvitz HR, Ruvkun G: The 21-nucleotide let-7 RNA regulates developmental timing in Caenorhabditis elegans. Nature 2000, 403:901-906.

17. Valoczi A, Hornyik C, Varga N, Burgyan J, Kauppinen S, Havelda Z: Sensitive and specific detection of microRNAs by northern blot analysis using LNA-modified oligonucleotide probes. Nucleic Acids Res 2004, 32:e175.

18. Berezikov E, Cuppen E, Plasterk RH: Approaches to microRNA discovery. Nat Genet 2006, 38(Suppl):S2-7.

19. Takada S, Berezikov E, Yamashita Y, Lagos-Quintana M, Kloosterman WP, Enomoto M, Hatanaka H, Fujiwara S, Watanabe H, Soda M, Choi YL, Plasterk RH, Cuppen E, Mano H: Mouse microRNA profiles determined with a new and sensitive cloning method. Nucleic Acids Res 2006, 34:e115.

20. Mineno J, Okamoto S, Ando T, Sato M, Chono H, Izu H, Takayama M, Asada $\mathrm{K}$, Mirochnitchenko O, Inouye M, Kato I: The expression profile of microRNAs in mouse embryos. Nucleic Acids Res 2006, 34:1765-1771.

21. Schmittgen TD, Jiang J, Liu Q, Yang L: A high-throughput method to monitor the expression of microRNA precursors. Nucleic Acids Res 2004, 32:e43.

22. Chen C, Ridzon DA, Broomer AJ, Zhou Z, Lee DH, Nguyen JT, Barbisin M, Xu NL, Mahuvakar VR, Andersen MR, Lao KQ, Livak KJ, Guegler KJ: Realtime quantification of microRNAs by stem-loop RT-PCR. Nucleic Acids Res 2005, 33:e179.

23. Cummins JM, He Y, Leary RJ, Pagliarini R, Diaz LA Jr, Sjoblom T, Barad O, Bentwich Z, Szafranska AE, Labourier E, Raymond CK, Roberts BS, Juhl H, Kinzler KW, Vogelstein B, Velculescu VE: The colorectal microRNAome. Proc Natl Acad Sci USA 2006, 103:3687-3692.

24. Lu J, Getz G, Miska EA, Alvarez-Saavedra E, Lamb J, Peck D, Sweet-Cordero A, Ebert BL, Mak RH, Ferrando AA, Downing JR, Jacks T, Horvitz HR, Golub TR: MicroRNA expression profiles classify human cancers. Nature 2005, 435:834-838.

25. Jay C, Nemunaitis J, Chen P, Fulgham P, Tong AW: miRNA profiling for diagnosis and prognosis of human cancer. DNA Cell Bio/ 2007, 26:293-300.

26. Yin JQ, Zhao RC, Morris KV: Profiling microRNA expression with microarrays. Trends Biotechnol 2008, 26:70-76.

27. Rosa A, Brivanlou AH: MicroRNAs in early vertebrate development. Cell Cycle 2009 in press.

28. Kloosterman WP, Plasterk RH: The diverse functions of microRNAs in animal development and disease. Dev Cell 2006, 11:441-450.

29. Asli NS, Pitulescu ME, Kessel M: MicroRNAs in organogenesis and disease. Curr Mol Med 2008, 8:698-710.

30. Esquela-Kerscher A, Slack FJ: Oncomirs: microRNAs with a role in cancer. Nature Rev Cancer 2006, 6:259-269.

31. Croce $C M$ : Causes and consequences of microRNA dysregulation in cancer. Nat Rev Genet 2009, 10:704-714.

32. Drakaki A, lliopoulos D: MicroRNA Gene Networks in Oncogenesis. Curr Genomics 2009, 10:35-41.

33. Latronico MV, Condorelli G: MicroRNAs and cardiac pathology. Nat Rev Cardiol 2009, 6:419-29.

34. Pauley KM, Chan EK: MicroRNAs and their emerging roles in immunology. Ann N Y Acad Sci 2008, 1143:226-239.

35. Bentwich I, Avniel A, Karov Y, Aharonov R, Gilad S, Barad O, Barzilai A, Einat P, Einav U, Meiri E, Sharon E, Spector Y, Bentwich Z: Identification of hundreds of conserved and nonconserved human microRNAs. Nat Genet 2005, 37:766-770.
36. Li SC, Pan CY, Lin WC: Bioinformatic discovery of microRNA precursors from human ESTs and introns. BMC Genomics 2006, 7:164.

37. Earley MC, Vogt RF Jr, Shapiro HM, Mandy FF, Kellar KL, Bellisario R, Pass KA, Marti GE, Stewart CC, Hannon WH: Report from a workshop on multianalyte microsphere assays. Cytometry 2002, 50:239-42.

38. Luminex xMAP Technology [http://www.luminexcorp.com/ technology/index.html]

39. Blenkiron C, Goldstein LD, Thorne NP, Spiteri I, Chin SF, Dunning MJ, Barbosa-Morais NL, Teschendorff AE, Green AR, Ellis IO, Tavaré S, Caldas C, Miska EA: MicroRNA expression profiling of human breast cancer identifies new markers of tumor subtype. Genome Biol 2007, 8:R214.

40. Taylor JD, Briley D, Nguyen Q, Long K, lannone MA, Li MS, Ye F, Afshari A, Lai E, Wagner M, Chen J, Weiner MP: Flow cytometric platform for highthroughput single nucleotide polymorphism analysis. BioTechniques 2001, 30:661-666. 668-669

41. Prabhakar U, Eirikis E, Davis HM: Simultaneous quantification of proinflammatory cytokines in human plasma using the LabMAP assay. Journal of immunological methods 2002, 260:207-218.

42. Mano H, Takada S: mRAP, a sensitive method for determination of microRNA expression profiles. Methods 2007, 43:118-122.

43. Takada S, Mano H: Profiling of microRNA expression by mRAP. Nature protocols 2007, 2:3136-3145.

44. Merlino G, Helman L: Rhabdomyosarcoma-working out the pathways. Oncogene 1999, 18:5340-348.

45. Shingara J, Keiger K, Shelton J, Laosinchai-Wolf W, Powers P, Conrad R, Brown D, Labourier E: An optimized isolation and labeling platform for accurate microRNA expression profiling. RNA 2005, 11:1461-1470.

46. Anderson J, Gordon T, McManus A, Mapp T, Gould S, Kelsey A, McDowell $H$, Pinkerton R, Shipley J, Pritchard-Jones K: Detection of the PAX3-FKHR fusion gene in paedriatic Rhabdomyosarcoma: a reproducible predictor of outcome? Br J Cancer 2001, 85:831-35.

47. De Pittà C, Tombolan L, Albiero G, Sartori F, Romualdi C, Jurman G, Carli M, Furlanello C, Lanfranchi G, Rosolen A: Gene expression profiling identifies potential relevant genes in alveolar rhabdomyosarcoma pathogenesis and discriminates $P A X 3-F K H R$ positive and negative tumors. Int J Cancer 2006, 118:2772-81.

48. Romualdi C, De Pittà C, Tombolan L, Bortoluzzi S, Sartori F, Rosolen A, Lanfranchi G: Defining the gene expression signature of rhabdomyosarcoma by meta-analysis. BMC Genomics 2006, 7:287.

49. Davicioni E, Finckenstein FG, Shahbazian V, Buckley JD, Triche TJ, Anderson MJ: Identification of a PAX-FKHR gene expression signature that defines molecular classes and determines the prognosis of alveolar rhabdomyosarcomas. Cancer Res 2006, 66:6936-46.

50. Romualdi C, Vitulo N, Del Favero M, Lanfranchi G: MIDAW: a web tool for statistical analysis of microarray data. Nucleic Acids Res 2005:W644-9.

51. Saeed Al, Bhagabati NK, Braisted JC, Liang W, Sharov V, Howe EA, Li J, Thiagarajan M, White JA, Quackenbush J: TM4 microarray software suite. Methods in Enzymology 2006, 411:134-93.

52. Tusher VG, Tibshirani R, Chu G: Diagnosis of multiple cancer types by shrunken centroids of gene expression. Proc Natl Acad Sci USA 2001, 98:5116-121.

53. Livak KJ, Schmittgen TD: Analysis of relative gene expression data using real-time quantitative PCR and the 2(-Delta Delta $C(T))$ Method. Methods 2001, 25:402-408.

doi: $10.1186 / 1471-2199-11-44$

Cite this article as: Biscontin et al., New miRNA labeling method for beadbased quantification BMC Molecular Biology 2010, 11:44 